\title{
Expression of LOXs and MMP-1, 2, 3 by ACL Fibroblasts and Synoviocytes Impact of Coculture and TNF- $\alpha$
}

\author{
Chunli Wang, PhD ${ }^{1}$ Qingjia Chi, $\mathrm{PhD}^{2}$ Chunming Xu, $\mathrm{PhD}^{1} \quad$ Kang Xu, $\mathrm{PhD}^{1} \quad$ Yanjun Zhang, $\mathrm{PhD}^{4}$ \\ Yan Liu, MS ${ }^{1}$ Li Yang, $\mathrm{PhD}^{1} \quad$ K. L. Paul Sung, $\mathrm{PhD}^{1,3}$ \\ 1 "111" Project Laboratory of Biomechanics and Tissue Repair, \\ Bioengineering College, Chongqing University, Chongqing, \\ Republic of China \\ 2 Department of Mechanics and Engineering Structure, Hubei Key \\ Laboratory of Theory and Application of Advanced Materials \\ Mechanics, Wuhan University of Technology, Wuhan, Hubei, \\ Republic of China \\ ${ }^{3}$ Department of Bioengineering and Orthopaedics, University of \\ California, San Diego, La Jolla, California \\ ${ }^{4}$ Department of Life Science, Hunan University of Science and \\ Technology, Xiangtan, Hunan, Republic of China \\ Address for correspondence K. L. Paul Sung, PhD, "111" Project \\ Laboratory of Biomechanics and Tissue Repair, College of \\ Bioengineering, Chongqing University, Chongqing 400044, \\ Republic of China (e-mail: klps@cqu.edu.cn).
}

J Knee Surg 2019;32:352-360.

\begin{abstract}
Keywords

- ACL

- synoviocytes

- MMPs

- LOXs

- coculture
\end{abstract}

Rupture of anterior cruciate ligament (ACL) has poor selfhealing ability. ${ }^{1}$ The gold standard of treatment for ACL injuries is reconstruction, but it remains difficult to acquire the satisfactory results and many of these patients may later develop knee osteoarthritis (OA). ${ }^{2}$ Limited self-healing capacity and unsatisfactory results of ACL reconstruction spur

received

September 19, 2017

accepted after revision

February 25, 2018

published online

April 10, 2018 interest to solve the mystery behind the mechanism of why the incomplete regeneration of injured $\mathrm{ACL}^{3,4} \mathrm{ACL}$ is an intraarticular but extrasynovial structure surrounded by a thin layer of synovial tissue within the knee joint environment. ${ }^{5}$ In the physiological articular joint, synovial membrane is thought to play a vital role in delivering nutrition and blood
Copyright $\odot 2019$ by Thieme Medical Publishers, Inc., 333 Seventh Avenue, New York, NY 10001, USA. Tel: +1(212) 584-4662.
DOI https://doi.org/ 10.1055/s-0038-1641592. ISSN 1538-8506. 
to ACL. ${ }^{6}$ Ruptured synovial tissue lead to the ACL exposed to synovial fluid involved in various kinds of proteolytic enzymes and cytokines which are mostly secreted by synovium. ${ }^{7}$ That means the synovium is considered to be the major regulator of the microenvironment in the joint cavity after ACL injury. Previous studies have revealed that the synovial fibroblasts played a major role in the wound healing of posterior cruciate ligament. ${ }^{8}$ However, it is much less understood whether the synoviocytes (SCs) influence the repair behavior of the ACL fibroblasts (ACLfs) in vitro model. Therefore, it would be important to develop a trans-well coculture system for defined studies of the crosstalk between the ACLfs and SCs to mimic the real knee microenvironment properly.

Besides direct depredation of the ACL extracellular matrix (ECM) via producing various proteolytic enzymes, the synovium could potently induce catabolic agents via stimulating the level of inflammatory cytokines. ${ }^{9,10}$ Irie et al have found an increased trend of the tumor necrosis factor- $\alpha$ (TNF- $\alpha$ ), interleukin-1 $\beta$ (IL-1 $\beta$ ), and IL- 6 in the joint fluid during the acute inflammation phase after ACL injury. ${ }^{11}$ These cytokines are also considered as important chemical mediators in the acute inflammatory phase of wound healing. ${ }^{12}$ TNF- $\alpha$ is one of the most important factors, and appears in the knee joint immediately after ligament injury and involve in wound healing mechanism through various ways. ${ }^{13}$ Previous studies have demonstrated that the ACLfs and synovial fibroblasts are very sensitive to TNF- $\alpha$ in regulating repair behavior of injured ACL. ${ }^{14,15}$ However, very few effects are known to the TNF- $\alpha$ responsiveness of ACLfs in SCs' coculture condition.

The anabolic elements of ligament healing include ECM production and modification, which is a key element of successful ligament healing, regulated by lysyl oxidases (LOXs). LOXs play an important role in the ECM synthesis, by catalyzing lysine-derived cross-links formation of the collagen and elastin. ${ }^{16}$ On the catabolic side, matrix metalloproteinases (MMPs) are capable of degrading almost all the components of ECM and play a vital role in tissue injury and repair process. ${ }^{17}$ Previous studies have revealed the relationship between TNF- $\alpha$ with LOXs and MMPs in many tissues. ${ }^{18}$ Our group also has indicated that higher expressions of MMPs, and relatively lower LOXs in ACLfs induced by TNF$\alpha$ compared with those in MCL fibroblasts in vitro monoculture condition, might explain the poor self-healing capacity of ACL. ${ }^{19}$ However, it remains unknown whether SCs regulate the LOXs and MMPs expression for ACLfs response to TNF- $\alpha$. Therefore, trans-well coculture system was used in this study to investigate the influence of cross-talking between ACLfs and SCS on the LOXs and MMP-1, 2, 3 expressions in ACLfs induced by TNF- $\alpha$.

\section{Materials and Methods}

\section{Cell Culture}

The human materials used for this study were obtained according to the ethical principles and the protocol was reviewed and approved by our Institutional Review Board (IRB).

The human ACLfs and SCs were respectively prepared from human ACL and synovial tissue (age range 30-60, four male and four female subjects) undergoing total knee replacement surgery after traffic accidents at the First Affiliated Hospital of Chongqing Medical University by means of the explants culture technique as described previous. We only guarantee that the samples had no inflammatory reaction in the knee joint caused by rheumatoid arthritis (RA), and no guarantee the samples had no inflammatory reaction in the knee joint caused by OA or long-term knee joint pathological changes. SCs from passage 1 to passage 3 and ACLfs from passage 3 to passage 6 were used in the experiments.

\section{Coculture}

With the Borden chamber (Corning, NY), the coculture model of ACLfs and SCs was established. The operating procedure has been described by previous study. ${ }^{20}$ Samples of culture media in the ACLfs were collected at 12, 24, 48, and 72 hours for MMP-2 activity assay by zymography. Samples of cell lysates in the ACLfs were collected at 1, 3,6, and 12 hours for LOXs and MMP-1, 2, 3 gene assay by quantitative reverse transcription polymerase chain reaction (qRT-PCR). Cell lysates samples in the ACLfs at 48 hours for LOXs and MMP-1, 2, 3 protein assay were collected by western blot.

\section{TNF- $\alpha$ Treatment}

Before being treated with recombinant human TNF- $\alpha(1,5$, $20 \mathrm{ng} / \mathrm{mL}$ ) (PeproTech, NJ), these ACLfs were cocultured with SCs as the same method with above. The steady-state levels of LOXs and MMP-1, 2, 3 messenger ribonucleic acid (mRNA) in cocultured ACLfs were determined for 12 hours after incubation in the presence or absence of TNF- $\alpha$. Coculture group without TNF- $\alpha$ treatment were used as controls. Then, we choose $5 \mathrm{ng} / \mathrm{mL}$ TNF- $\alpha$ for time course experiments, and this concentration was used throughout this study. The gene expression levels of LOXs and MMPs were detected by qRTPCR at 1, 3, 6, and 12 hours. The protein expression of LOXs and MMPs were analyzed by western blot at 48 hours.

\section{Mechanical Injury}

The Flexercell Tension Plus system 4000 (Flexcell International Corp., Hillsborough, CA) was used in the study, and $12 \%$ mechanical stretch (traumatic rupture) to the ACLfs was applied. ${ }^{20}$ The gene expression levels of LOXs and MMPs were detected by qRT-PCR at 1, 3, 6, and 12 hours. The protein expression of LOXs and MMPs were analyzed by western blot at 48 hours.

\section{Quantitative Real-Time Polymerase Chain Reaction}

Quantitative real-time PCR was performed with QuantiTect SYBR Green PCR kit (Qiagen, German) using iCycler (BioRad) according to the operation procedure. Quantitative real-time PCR reactions were performed at $0.5 \mu \mathrm{M}$ for each primer ( - Table 1 ) in a 25 - $\mu \mathrm{L}$ volume containing $1 \mu \mathrm{L}$ complementary deoxyribonucleic acid (cDNA) sample.

\section{Western Blotting}

LOXs and MMP-1, 2, 3 protein level were assayed from the collected lysates samples by western blot as described previously. ${ }^{21}$ 
Table 1 Primers of housekeeping gene (GAPDH), lysyl oxidase family (LOXs), and matrix metalloproteinases (MMP-1, 2, 3) designed for quantitative real-time PCR

\begin{tabular}{|l|l|}
\hline mRNA & Primer pairs \\
\hline GAPDH (138 bp) & $\begin{array}{l}\text { Forward GCACCGTCAAGGCTGAGAAC } \\
\text { Reverse TGGTGAAGACGCCAGTGGA }\end{array}$ \\
\hline LOX (183 bp) & $\begin{array}{l}\text { Forward GCATACAGGGCAGATGTCAGA } \\
\text { Reverse TTGGCATCAAGCAGGTCATAG }\end{array}$ \\
\hline LOXL-1 (135 bp) & $\begin{array}{l}\text { Forward TGCCACCAGCATTACCACAG } \\
\text { Reverse GAGGTTGCCGAAGTCACAGG }\end{array}$ \\
\hline LOXL-2 (149 bp) & $\begin{array}{l}\text { Forward CTGCAAGTTCAATGCCGAGT } \\
\text { Reverse TCTCCACCAGCACCTCCACTC }\end{array}$ \\
\hline LOXL-3 (146 bp) & $\begin{array}{l}\text { Forward CAACAGGAGGTTTGAACGCTAC } \\
\text { Reverse GCTGACATGGGTTTCTTGGTAA }\end{array}$ \\
\hline LOXL-4 (155 bp) & $\begin{array}{l}\text { Forward TTCACCCACTACGACCTCCTCA } \\
\text { Reverse CAGCAGCCTACAGTCACTCCCT }\end{array}$ \\
\hline MMP-1 (170 bp) & $\begin{array}{l}\text { Forward GCTGAAAGTGACTGGGAAACC } \\
\text { Reverse TGCTCTTGGCAAATCTGGCGTG }\end{array}$ \\
\hline MMP-2 (179 bp) & $\begin{array}{l}\text { Forward GTGACGGAAAGATGTGGTG } \\
\text { Reverse GGTGTAGGTGTAAATGGGTG }\end{array}$ \\
\hline MMP-3 (122 bp) & $\begin{array}{l}\text { Forward GACAAAGGATACAACAGGGAC } \\
\text { Reverse TGAGTGAGTGATAGAGTGGG }\end{array}$ \\
\hline
\end{tabular}

Abbreviations: bp, base pair; GAPDH, glyceraldehyde 3-phosphate dehydrogenase; mRNA, messenger ribonucleic acid; PCR, polymerase chain reaction.

\section{Zymography}

MMP-2 activity was assayed from the collected culture media samples using $0.05 \%$ gelatin zymography as described previously. $^{1}$

\section{Statistical Analysis}

Data are expressed as mean \pm standard deviation (SD). Statistical analysis was performed by one-way analysis of variance (ANOVA). Post hoc analysis utilized Fisher's least significant difference (LSD). In each analysis, a value of $p<0.05$ was accepted as statistically significant.

\section{Results}

\section{TNF- $\alpha$ Induced Concentration-Dependent Decrease of the LOXs and Increase of MMP-1, 2, 3 in Cocultured ACLfs}

Here, we first detected the effects of TNF- $\alpha$ on the gene expression of the LOXs and MMP-1, 2, 3 in ACLfs after SCs coculture. Quantitative real-time PCR ( - Fig. 1A) showed that TNF- $\alpha$ led to decreases in LOXs expressions that were concentration dependent. The inhibitory effect reached a maximum at $20 \mathrm{ng} / \mathrm{mL}$ TNF- $\alpha$ for all LOXs family. TNF- $\alpha$ at 20ng/mL decreased LOX mRNA levels to 71\%; LOXL-1 to $63 \%$; LOXL-2 to 54\%; LOXL-3 to $47 \%$; and LOXL-4 to $28 \%$, respectively, in cocultured ACLfs. TNF- $\alpha$ at all concentrations significantly increased MMP-1, 2, 3 expressions (-Fig. 1B). The stimulation effect reached a maximum (2.31-, 1.89-, and 2.52 -fold, respectively) at $20 \mathrm{ng} / \mathrm{mL}$ TNF- $\alpha$ for MMP-1, $2,3$. After treated with 1,5 , and $20 \mathrm{ng} / \mathrm{mL}$ TNF- $\alpha$, MMP-2 activity both in monoculture and coculture ACLfs were significantly promoted in a dose-dependent manner compared with the control group (the monoculture group without TNF- $\alpha$ ). Besides, MMP-2 activities could be enhanced in the coculture groups compared with the monoculture groups (-Fig. 1C,D).

\section{Regulation of LOXs Expression in Cocultured ACLfs by TNF- $\alpha$}

Coculture alone induced the increase of LOXs mRNA in ACLfs compared with the monoculture (control). However, TNF- $\alpha$ alone and combination of coculture significantly inhibit the gene expression of LOXs in time-dependent manner. To some extent, the coculture further promotes the TNF- $\alpha$ to induce the decrease of LOXs in the transcription level. The inhibitory effect of TNF- $\alpha$ on the gene expression of LOXs in monoculture ACLfs decreased to a minimum (0.83-, 0.68-, 0.45-, $0.51-$, and 0.39 -fold, respectively) at 12 hours. The mRNA level of LOX and LOXL1-4 were respectively decreased by $0.62-, 0.50-, 0.52-, 0.51-$, and 0.47 -fold at 12 hours in coculture ACLfs induced by TNF- $\alpha$ versus control (-Fig. 2A-E). After 48-hour treatment, the TNF- $\alpha$ and combination of coculture significantly reduced the protein level of LOXs in normal ACLfs. The bands of LOXs showed that the optical density in the coculture, TNF- $\alpha$, and cotreatment groups were lower than control (-Fig. 2F) and further semiquantification confirmed the same (-Fig. 2G).

\section{Regulation of MMP-1, 2, 3 Expression in Cocultured ACLfs by TNF- $\alpha$}

From the - Fig. $\mathbf{3 A}-\mathbf{C}$, we found that coculture alone slightly increases the expression of MMP-1, 2, 3 mRNA compared with the monoculture (control). TNF- $\alpha$ also upregulated the gene expression of MMP-1, 2, and 3 compared with the control ( - Fig. 3A-C). The MMP-2 and MMP-3 reached a maximum at 6 hours (increase of 2.13- and 3.45-fold, respectively). The gene expression of MMP-1 peaked at 12 hours and increased by 3.25-fold. The combination of TNF- $\alpha$ and SCs coculture induced higher increases of MMP-1, 2, 3 than that of coculture alone without TNF- $\alpha$ and ACLf treated with TNF- $\alpha$ alone at 12 hours. From - Fig. 3D,E, we found that results of protein in MMP-1, 2, 3 remain the same with the results of gene.

\section{TNF- $\alpha$ Induced Decrease of LOXs in Cocultured ACLfs after Mechanical Injury}

Mechanical injury alone upregulated the mRNA levels of LOXs in monoculture ACLfs within 24 hours compared with control (data have been published by our group ${ }^{22}$ ). In presence of TNF- $\alpha$, the results showed mechanical injury significantly inhibit the gene expression of LOXs at each time point. This inhibitory effect decreased to a minimum at 12 hours (0.62-, 0.53-, 0.66-, 0.63-, and 0.47-fold, respectively, for LOX and LOXL1-4) compared with control values. Coculture further decreased the gene expression of LOXs below control in injured ACLfs treated with TNF- $\alpha$. The expression of LOX and LOXL1-4 were decreased to $38 \%, 25 \%, 14 \%, 22 \%$, and $10 \%$ at 12 hours, respectively ( - Fig. 4A-E). The results from the western blot analysis demonstrated that the combination of injury and TNF- $\alpha$ treatment led to a decrease in the LOXs (except for LOXL-2) 

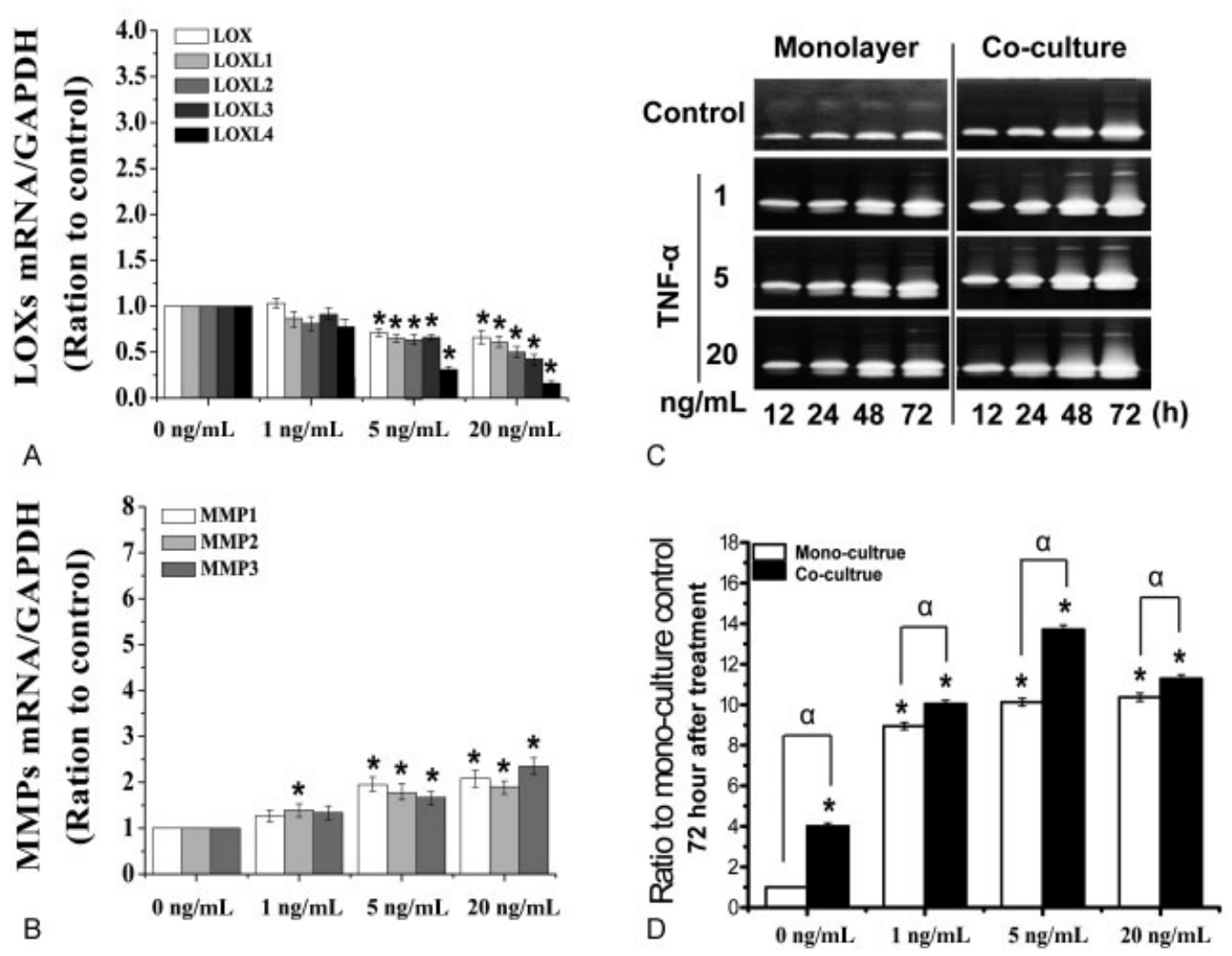

Fig. 1 Effects of tumor necrosis factor- $\alpha(T N F-\alpha)$ with different concentrations $(0-20 \mathrm{ng} / \mathrm{mL})$ on the expressions of messenger ribonucleic acid (mRNA) for lysyl oxidase (LOX) family and matrix metalloproteinase (MMP)-1, 2, 3 in normal anterior cruciate ligament fibroblasts (ACLfs) after synoviocytes (SCs) coculture. (A) The LOX family gene expressions. (B) The MMP-1, 2, 3 gene expressions. Data (mean \pm SD, $n=3$ ) were represented as the fold change in expression compared with control. (C) The MMP-2 activity in both monolayer and coculture ACLfs in presence of TNF- $\alpha(1,5$, and $20 \mathrm{ng} / \mathrm{mL})$. The gels shown were representative of three different experiment $(n=3)$. (D) Quantitative analysis of MMP-2 activity at 72 hours with the Integrated Performance Primitives (IPP) software. The data were the mean of three different experiments $(n=3)$. Statistical analysis was done by analysis of variance (ANOVA). ${ }^{*}$ Significant difference with respect to control $(p<0.05)$.

expression in both monoculture and coculture ACLfs at 48 hours (-Fig. 4F). The semiquantification showed that the LOX and LOXL1-4 synthesis were downregulated by 0.15-, 0.20-, 1.7-, $0.46-$, and 0.45 -fold, respectively, in coculture ACLfs treated with TNF- $\alpha$ (-Fig. 4G).

\section{TNF- $\alpha$ Induced Overexpression of MMP-1, 2, 3 in Cocultured ACLfs after Mechanical Injury}

Mechanical injury alone upregulated the mRNA expression of MMP-1, 2, 3 in monoculture ACLfs (data have been published by our group ${ }^{22}$ ). The combination of mechanical injury and SCs coculture induced higher increases of MMP-1, 2, 3 than that of co-culture alone without injury and ACLf treated with injury alone at 12 hours (-Fig. 5A-C). The MMP-2 mRNA level only was slightly promoted at 6 hours in the coculture ACLfs after mechanical injury. TNF- $\alpha$ significantly upregulated the gene expression of MMP-1, $-2,-3$ in injured ACLfs both in the absence of and presence of SCs (-Fig. $\mathbf{5 A}-\mathbf{C}$ ). The coculture further promoted that the TNF- $\alpha$ induced the increase of MMP-1, 2, 3 in the injured ACLfs. The level of MMP- 1 and -3 reached a maximum at 6 hours (increase of 5.87- and 4.16-fold in monoculture and 8.13- and 9.15-fold at 12 hours in coculture, respectively). From - Fig. 5D and E, we found that results of protein for 48 hours in MMP-1, 2, 3 remain the same with the results of gene. In the injured monolayer group, TNF- $\alpha$ and coculture promoted ACLfs to express much more MMP-2 than injury controls and the coculture had a greater influence on MMP-2 activity than TNF- $\alpha$ ( - Fig. $\mathbf{5 F}$ and $\mathbf{G}$ ). In the injured coculture group, the $72 \mathrm{kDa}$ pro-form and the $62 \mathrm{kDa}$ active-form of MMP2 significantly increased after TNF- $\alpha$ induction (-Fig. 5F).

\section{Discussion}

The most important finding of this study was that SCs coculture significantly affected the ACL fibroblastic response to a combination of high mechanical strain and TNF- $\alpha$. A major reason for the failure to self-healing of $\mathrm{ACL}$ is the inadequate knowledge of their complicated environment and it is difficult to correlate what is studied in the laboratory setting to what actually occurs during an ACL injury event. Previous study had proved that synovium involved in regulating the microenvironment of joint cavity. ${ }^{5}$ However, little is known about whether the SCs affect the repair behaviors of the ACLfs and its contribution to the ECM degradation of the ACLfs in in vitro model. The in vitro coculture model has been used in our laboratory, providing a feasible way to mimic the real situation in vivo, to understand molecular mechanisms of cell-cell interactions. ${ }^{23}$ This coculture system also has been used 

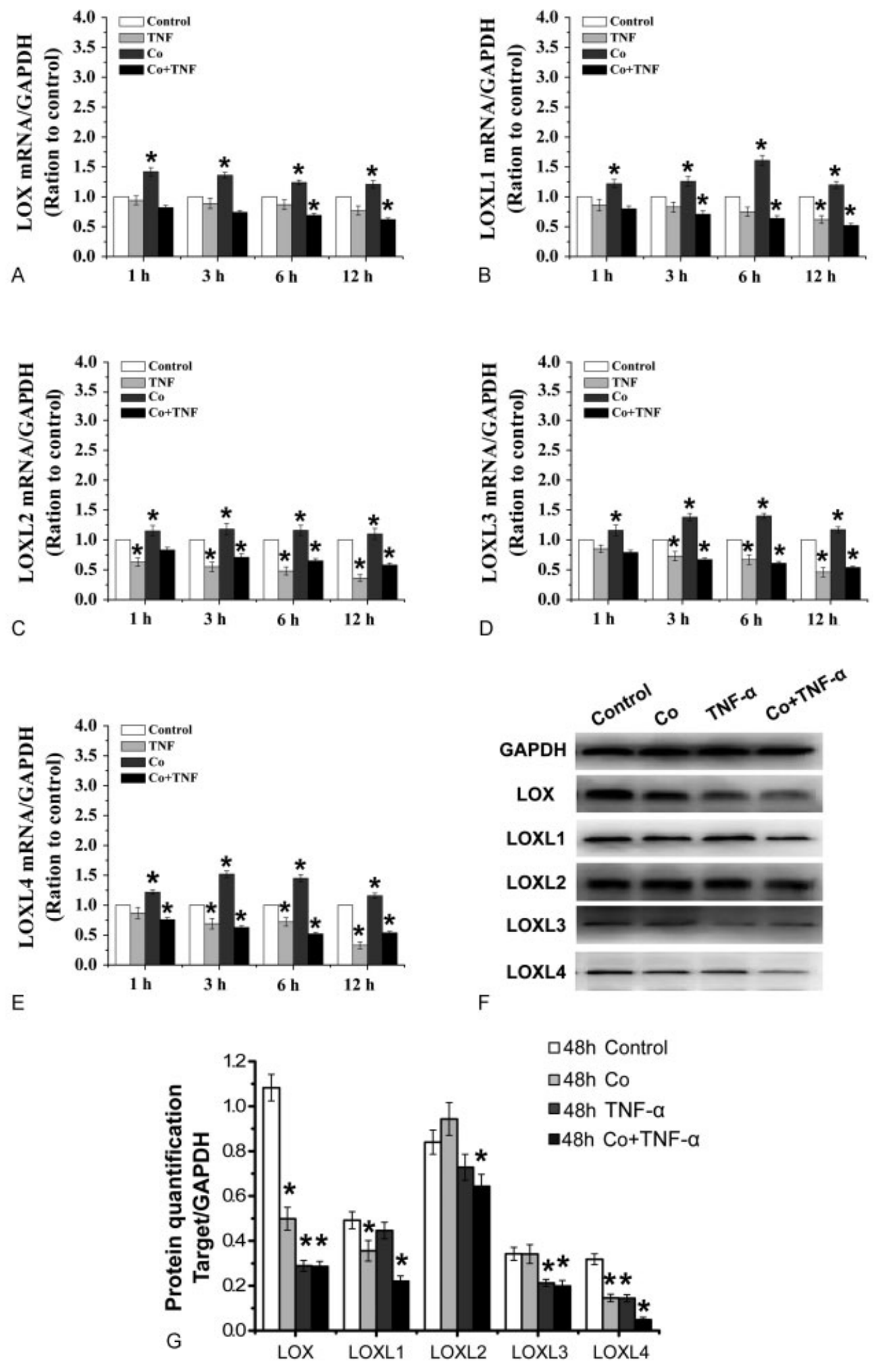

Fig. 2 Effects of tumor necrosis factor- $\alpha(T N F-\alpha)$ on lysyl oxidases (LOXs) gene and protein expression in normal anterior cruciate ligament fibroblasts (ACLfs) in both monoculture and coculture condition. (A-E). The LOXs gene expression. Data (mean \pm SD, $n=3$ ) were represented as the fold change in expression compared with control. (F) The LOXs protein expression. Glyceraldehyde 3-phosphate dehydrogenase (GAPDH) served as an internal control. The bands shown were representative of three different experiment $(n=3)$. (G) Quantitative analysis of LOXs synthesis in ACLfs at 48 hours with the Integrated Performance Primitives (IPP) software. The data were the mean of three different experiments $(n=3)$. Statistical analysis was done by analysis of variance (ANOVA). * Significant difference with respect to control $(p<0.05)$. 

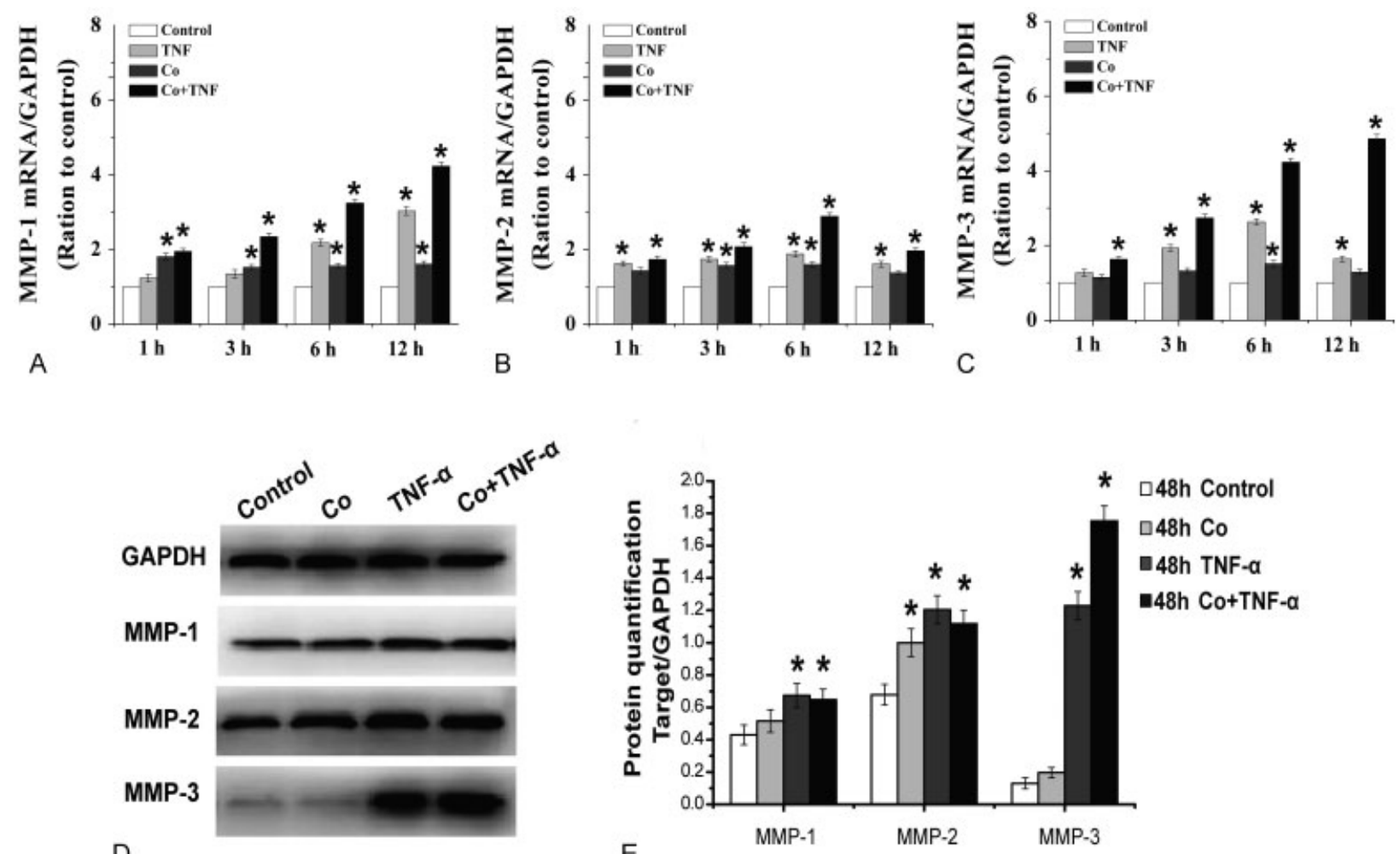

D

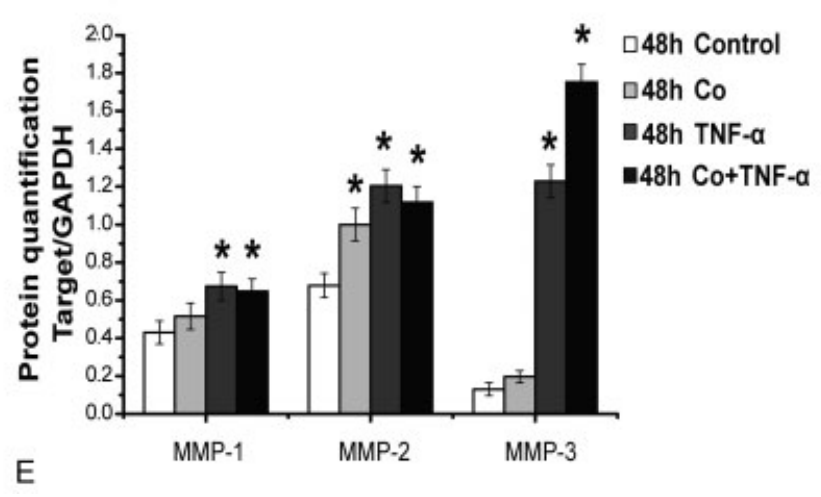

Fig. 3 Effects of tumor necrosis factor- $\alpha(T N F-\alpha)$ on matrix metalloproteinase (MMP)-1, 2, 3 gene and protein expression in normal anterior cruciate ligament fibroblasts (ACLfs) in both monoculture and coculture condition. (A-C) The MMP-1, 2, 3 gene expression. Data (mean \pm SD, $n=3$ ) were represented as the fold change in expression compared with control. (D) The MMP-1, 2, 3 protein expression. Glyceraldehyde 3phosphate dehydrogenase (GAPDH) served as an internal control. The bands shown were representative of three different experiment $(n=3)$. (E) Quantitative analysis of MMP-1, 2, 3 protein in ACLfs at 48 hour with the Integrated Performance Primitives (IPP) software. The data were the mean of three different experiments $(n=3)$. Statistical analysis was done by analysis of variance (ANOVA). ${ }^{*}$ Significant difference with respect to control $(p<0.05)$.

widely by other laboratories to investigate the importance of cocultures with different cell types to induce various desired effects on cell differentiation, cell activities, cell death, and proliferative properties due to reciprocal influences. ${ }^{24}$ Based on the previous reports, the SCs were used to coculture with ACLfs to mimic the synovial fluid microenvironment and to detect how the SCs affect the repair behaviors of the ACLfs. The slight increase of LOXs and MMP-1, 2, 3 gene expression within 12 hours may be a ECM homeostatic balance process modified by the crosstalk between ACLfs and SCs. The protein expression of MMP-1, 2, 3 and LOXs were unchanged in ACLfs after coculture at 48 hours. The results demonstrated that the SCs had a minor effect on the normal ACLfs.

Inflammation is the initial response to ACL injury. Previous studies revealed that both the ACLfs and SCs are very susceptible to TNF- $\alpha$ in regulating the expressions of MMPs and LOXs. ${ }^{8,14}$ TNF- $\alpha$ could destroy the balance of ECM through various ways, including inhibition of LOXs expression in the ACLf, promotion of MMPs expression in the ACLfs, and perisurrounded SCs. Previous data have proved that both ACLfs and SCs in the presence of TNF- $\alpha$ has high MMP activities, which have been hypothesized to primarily be responsible for the poor self-healing of cruciate ligaments in monoculture condition. ${ }^{21}$ It is unclear whether the SCs could affect the regulation of TNF- $\alpha$ on expression of LOXs and MMPs in ACLfs. This study directly demonstrates that there are no significant differences in the inhibitory effect of TNF- $\alpha$ on the LOXs gene and protein expression between the normal monoculture and coculture ACLfs. However, the high levels of MMP-1, 2, 3 in normal ACLfs induced by TNF- $\alpha$ are further promoted by coculture.

In the real physiological situation, the inflammatory cytokine TNF- $\alpha$, which accumulates immediately after ACL injury and remains there for approximately 1 week, may exert its effect together with the stretch forces to induce the injurious cascade. The application of $12 \%$ mechanical stretch to ACLfs are considered pathologic strains in in vitro model in this experiment. ${ }^{25}$ The observed decrease of LOXs and the overexpression of MMP-1, 2, 3 by pathologic stimuli in coculture ACLfs are consistent with other in vitro studies in various types of cells such as posterior cruciate ligament. ${ }^{20}$ The consistency proved that the SCs strongly modified homeostatic balance of ECM and appeared to have a significant impact on cruciate ligament wound healing. Moreover, in the pathologic strains condition, the SCs further promote the regulation of TNF- $\alpha$ on both the LOXs and MMPs. The results showed that the SCs coculture further enhanced the TNF- $\alpha$-induced upregulation of MMPs expressions in injured ACLfs. Contrary to MMPs, the decrease 

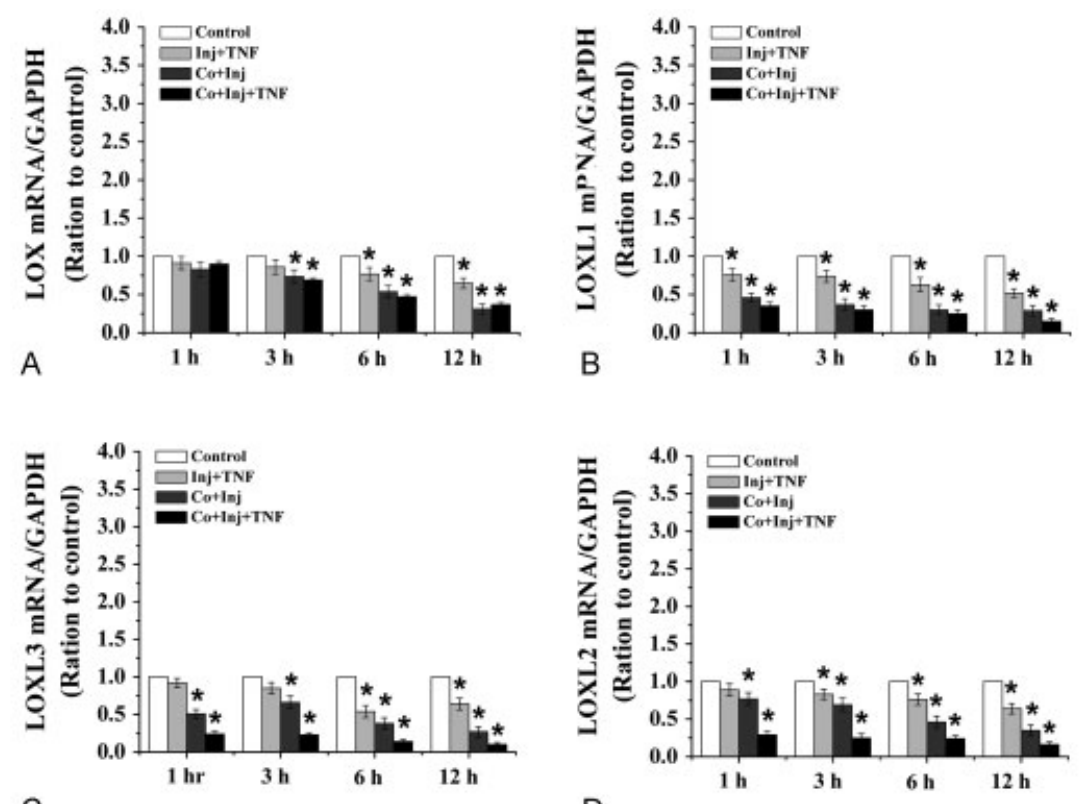

C

D
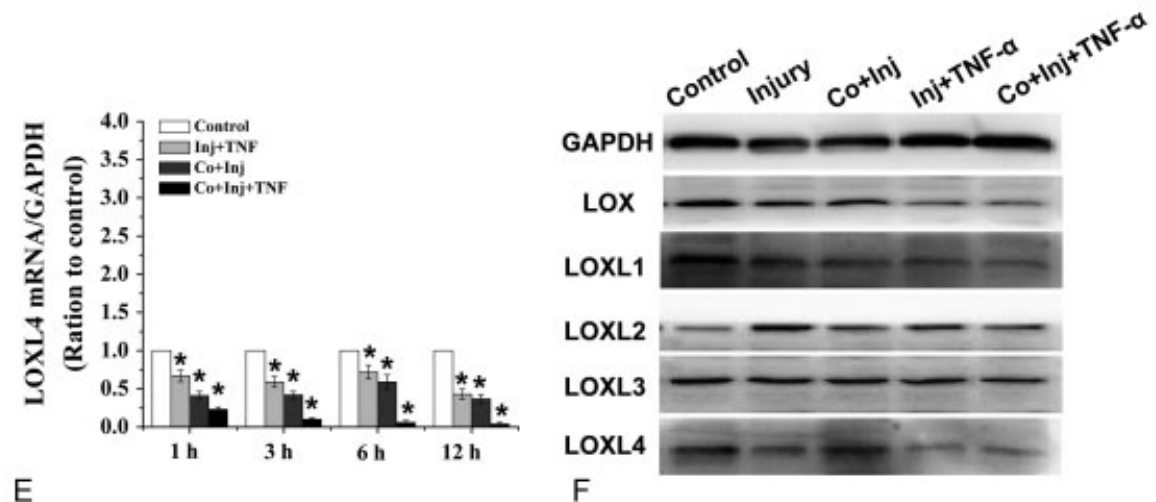

$\mathrm{E}$

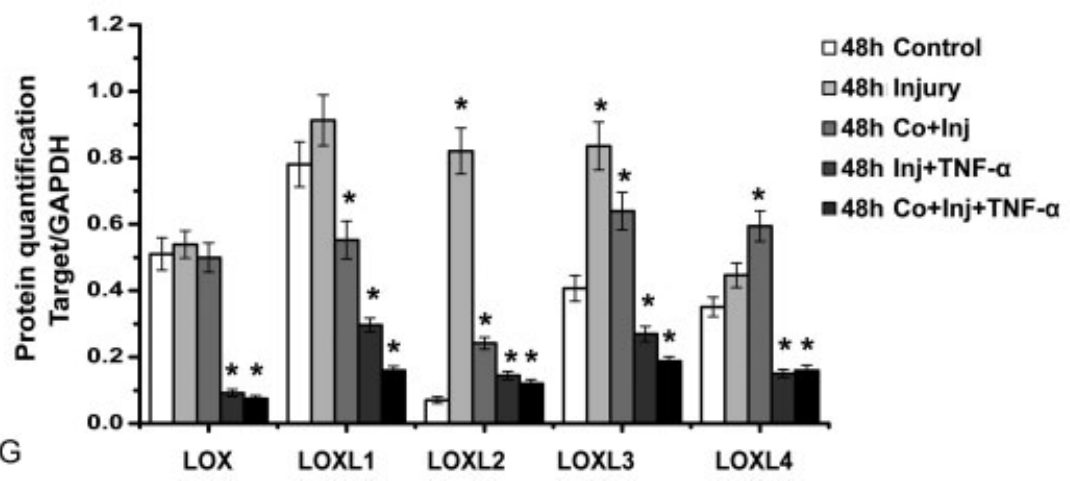

Fig. 4 Effects of tumor necrosis factor- $\alpha(T N F-\alpha)$ on lysyl oxidases (LOXs) messenger ribonucleic acid (mRNA) and protein expression in injured anterior cruciate ligament fibroblasts (ACLf) in both monoculture and coculture condition. (A-E) The LOXs gene expression. Data (mean \pm SD, $n=3$ ) were represented as the fold change in expression compared with control. (F) The LOXs protein expression. Glyceraldehyde 3-phosphate dehydrogenase (GAPDH) served as an internal control. The bands shown were representative of three different experiment $(n=3)$. (G) Quantitative analysis of LOXs synthesis in injured ACLfs at 48 hours with the Integrated Performance Primitives (IPP) software. The data were the mean of three different experiments $(n=3)$. Statistical analysis was done by analysis of variance (ANOVA). ${ }^{*}$ Significant difference with respect to control $(p<0.05)$.

of LOXs in injured ACLfs by TNF- $\alpha$ was further inhibited by SCs coculture. Based on these results, we postulate that the seeded SCs will attach to the semipermeable membrane and release some cytokines which facilitates the ability of TNF- $\alpha$ to activate each other and create a complex network of proteases in the ACLfs. The overrelease of MMPs not only depredate ECM of ACL to restrict the regeneration and repair but also increase cartilage degradation and accelerate $\mathrm{OA}$ in injured joint. Multiple studies have described the promotion of TNF- $\alpha$ to expressions of MMPs in synovium, which contributes to the development of inflammatory disease such as chronic wounds, RA, and OA. ${ }^{26}$ 

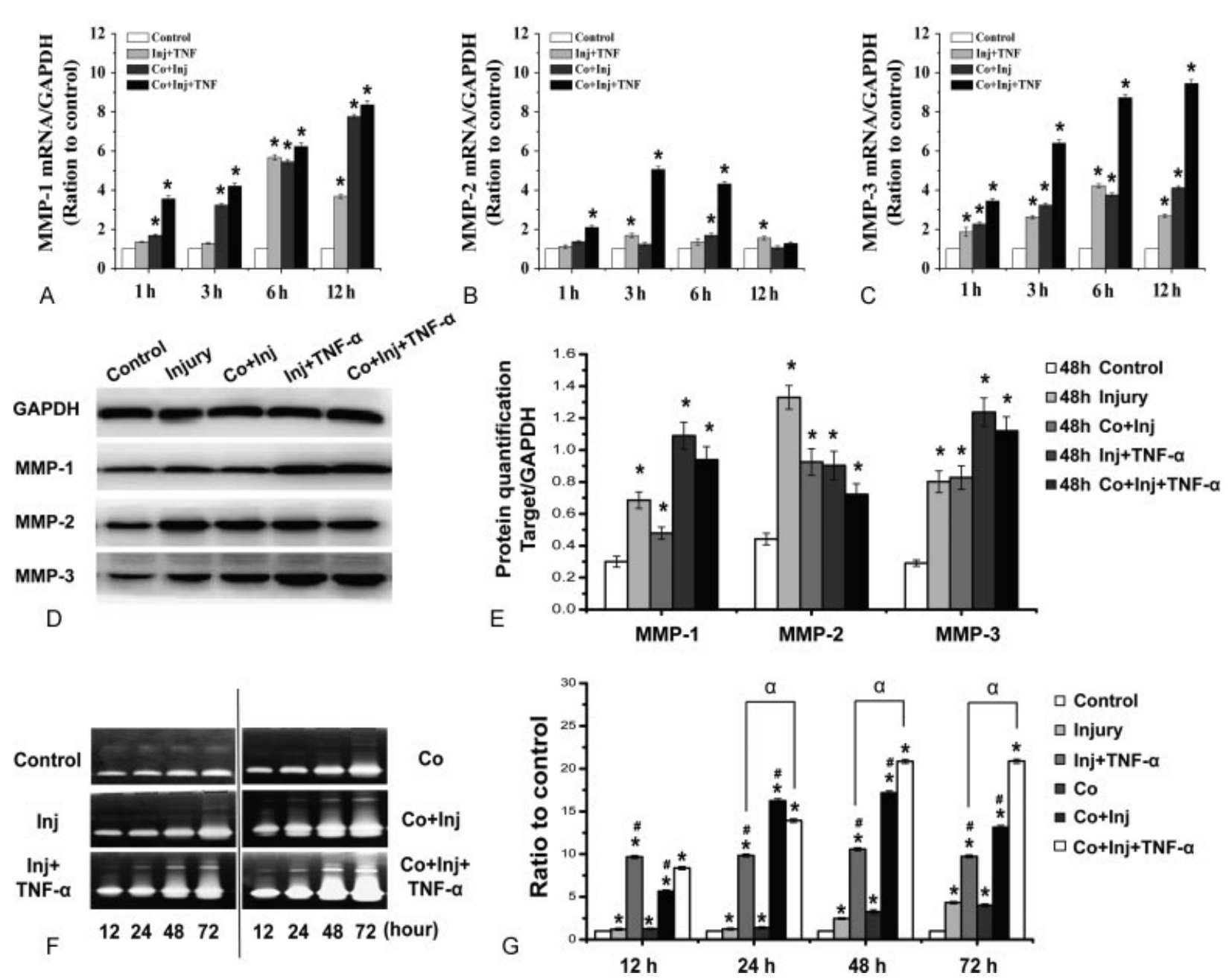

Fig. 5 Effects of tumor necrosis factor- $\alpha(T N F-\alpha)$ on matrix metalloproteinase (MMP)-1, 2, 3 messenger ribonucleic acid (mRNA) and protein expression in injured anterior cruciate ligament fibroblasts (ACLfs) in absence and presence of coculture condition. (A-C) The MMP-1, 2, 3 gene expression. Data (mean $\pm S D, n=3$ ) were represented as the fold change in expression compared with control. (D) The MMP-1, 2, 3 protein expression. Glyceraldehyde 3-phosphate dehydrogenase (GAPDH) served as an internal control. The bands shown were representative of three different experiment $(n=3)$. (E) Quantitative analysis of MMP-1, 2, 3 protein in injured ACLfs at 48 hours with the Integrated Performance Primitives (IPP) software. The data were the mean of three different experiments $(n=3)$. (F) The MMP-2 activity in injured ACLfs treated with TNF- $\alpha$ in both the monoculture and coculture groups. The gels shown were representative of three different experiment $(n=3)$. (G) Quantitative analysis of MMP-2 activity at 12, 24, 48, and 72 hours with the IPP software. The data were the mean of three different experiments $(n=3)$. Statistical analysis was done by analysis of variance (ANOVA). *Significant difference with respect to control $(p<0.05)$. \#Significant difference with respect to injury group $(p<0.05)$.

The results showed SCs could significantly affect the regulatory effect of TNF- $\alpha$ on the anabolic and catabolic gene and protein expression in ACLfs. It was confirmed in vitro that the synovium tissue is another potential reason for the poor self-healing capacity of injured ACL ligament in vitro.

\section{Conflict of Interest}

None.

\section{Reference}

1 Zhou D, Lee HS, Villarreal F, et al. Differential MMP-2 activity of ligament cells under mechanical stretch injury: an in vitro study on human ACL and MCL fibroblasts. J Orthop Res 2005;23(04):949-957
2 Lohmander LS, Englund PM, Dahl LL, Roos EM. The long-term consequence of anterior cruciate ligament and meniscus injuries: osteoarthritis. Am J Sports Med 2007;35(10):1756-1769

3 Sung KL, Yang L, Whittemore DE, et al. The differential adhesion forces of anterior cruciate and medial collateral ligament fibroblasts: effects of tropomodulin, talin, vinculin, and alpha-actinin. Proc Natl Acad Sci U S A 1996;93(17):9182-9187

4 Nagineni CN, Amiel D, Green MH, Berchuck M, Akeson WH. Characterization of the intrinsic properties of the anterior cruciate and medial collateral ligament cells: an in vitro cell culture study. J Orthop Res 1992;10(04):465-475

5 Tang Z, Yang L, Wang Y, et al. Contributions of different intraarticular tissues to the acute phase elevation of synovial fluid MMP-2 following rat ACL rupture. J Orthop Res 2009;27(02):243-248

6 Kiapour AM, Murray MM. Basic science of anterior cruciate ligament injury and repair. Bone Joint Res 2014;3(02):20-31

7 Barlow Y, Willoughby J. Pathophysiology of soft tissue repair. $\mathrm{Br}$ Med Bull 1992;48(03):698-711 
8 Zhang Y, Huang W, Jiang J, et al. Influence of TNF- $\alpha$ and biomechanical stress on matrix metalloproteinases and lysyl oxidases expressions in human knee synovial fibroblasts. Knee Surg Sports Traumatol Arthrosc 2014;22(09):1997-2006

9 Wang Y, Tang Z, Xue R, et al. Combined effects of TNF- $\alpha$, IL-1 $1 \beta$, and HIF- $1 \alpha$ on MMP- 2 production in ACL fibroblasts under mechanical stretch: an in vitro study. J Orthop Res 2011;29(07):1008-1014

10 Chamberlain CS, Brounts SH, Sterken DG, Rolnick KI, Baer GS, Vanderby R. Gene profiling of the rat medial collateral ligament during early healing using microarray analysis. J Appl Physiol (1985) 2011;111(02):552-565

11 Irie K, Uchiyama E, Iwaso H. Intraarticular inflammatory cytokines in acute anterior cruciate ligament injured knee. Knee 2003; 10(01):93-96

12 Xie J, Wang C, Yin L, Xu C, Zhang Y, Sung KL. Interleukin-1 beta influences on lysyl oxidases and matrix metalloproteinases profile of injured anterior cruciate ligament and medial collateral ligament fibroblasts. Int Orthop 2013;37(03):495-505

13 Greenwel P, Tanaka S, Penkov D, et al. Tumor necrosis factor alpha inhibits type I collagen synthesis through repressive CCAAT/ enhancer-binding proteins. Mol Cell Biol 2000;20(03):912-918

14 Xie J, Jiang J, Huang W, et al. TNF- $\alpha$ induced down-regulation of lysyl oxidase family in anterior cruciate ligament and medial collateral ligament fibroblasts. Knee 2014;21(01):47-53

15 Wang Y, Yang L, Zhang J, et al. Differential MMP-2 activity induced by mechanical compression and inflammatory factors in human synoviocytes. Mol Cell Biomech 2010;7(02):105-114

16 Kagan HM, Li W. Lysyl oxidase: properties, specificity, and biological roles inside and outside of the cell. J Cell Biochem 2003;88(04):660-672

17 Steffensen B, Häkkinen L, Larjava H. Proteolytic events of woundhealing-coordinated interactions among matrix metalloproteinases (MMPs), integrins, and extracellular matrix molecules. Crit Rev Oral Biol Med 2001;12(05):373-398
18 Pahuja N, Kumar NR, Shroff R, et al. Differential molecular expression of extracellular matrix and inflammatory genes at the corneal cone apex drives focal weakening in keratoconus. Invest Ophthalmol Vis Sci 2016;57(13):5372-5382

19 Cai L, An S, Liao J, et al. Influences of tumor necrosis factor- $\alpha$ on lysyl oxidases and matrix metalloproteinases of injured anterior cruciate ligament and medial collateral ligament fibroblasts. J Knee Surg 2017;30(01):78-87

20 Wang C, Xie J, Jiang J, et al. Differential expressions of the lysyl oxidase family and matrix metalloproteinases-1, 2, 3 in posterior cruciate ligament fibroblasts after being co-cultured with synovial cells. Int Orthop 2015;39(01):183-191

21 Xie J, Wang CL, Yang W, et al. Modulation of MMP-2 and MMP-9 through connected pathways and growth factors is critical for extracellular matrix balance of intra-articular ligaments. J Tissue Eng Regen Med 2016. Doi: 10.1002/term.2325

22 Xie J, Wang C, Huang DY, et al. TGF-beta 1 induces the different expressions of lysyl oxidases and matrix metalloproteinases in anterior cruciate ligament and medial collateral ligament fibroblasts after mechanical injury. J Biomech 2013;46(05): 890-898

23 Suzuki K, Saito J, Yanai R, et al. Cell-matrix and cell-cell interactions during corneal epithelial wound healing. Prog Retin Eye Res 2003;22(02):113-133

24 Wang HQ, Bai L, Shen BR, Yan ZQ, Jiang ZL. Coculture with endothelial cells enhances vascular smooth muscle cell adhesion and spreading via activation of $\beta 1$-integrin and phosphatidylinositol 3-kinase/Akt. Eur J Cell Biol 2007;86(01):51-62

25 Fleming BC, Beynnon BD. In vivo measurement of ligament/ tendon strains and forces: a review. Ann Biomed Eng 2004;32 (03):318-328

26 Hou WS, Li Z, Gordon RE, et al. Cathepsin k is a critical protease in synovial fibroblast-mediated collagen degradation. Am J Pathol 2001;159(06):2167-2177 